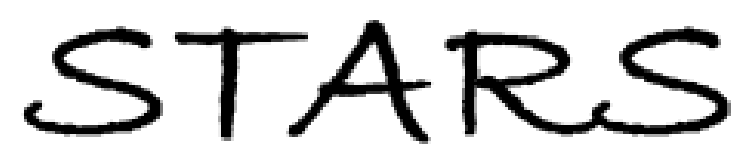

University of Central Florida

STARS

$1-1-2004$

\title{
Model of wide-angle optical field propagation using scalar diffraction theory
}

Vesselin Shaoulov

University of Central Florida

Ravi Vijaya Satya

University of Central Florida

Guy Schiavone

University of Central Florida

Eric Clarkson

Jannick Rolland

University of Central Florida

Find similar works at: https://stars.library.ucf.edu/facultybib2000

University of Central Florida Libraries http://library.ucf.edu

This Article is brought to you for free and open access by the Faculty Bibliography at STARS. It has been accepted for inclusion in Faculty Bibliography 2000s by an authorized administrator of STARS. For more information, please contactSTARS@ucf.edu.

\section{Recommended Citation}

Shaoulov, Vesselin; Satya, Ravi Vijaya; Schiavone, Guy; Clarkson, Eric; and Rolland, Jannick, "Model of wide-angle optical field propagation using scalar diffraction theory" (2004). Faculty Bibliography 2000 s. 4791.

https://stars.library.ucf.edu/facultybib2000/4791

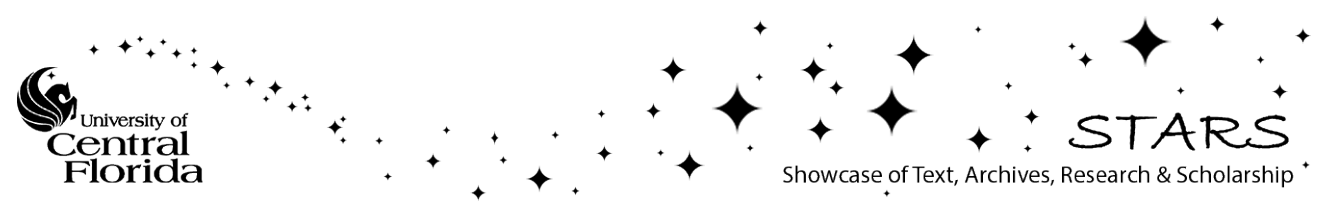




\section{Model of wide-angle optical field propagation using scalar diffraction theory}

\author{
Vesselin Shaoulov, MEMBER SPIE \\ University of Central Florida \\ School of Optics \\ Center for Research and Education in \\ Optics and Lasers \\ Florida Photonics Center of Excellence \\ Orlando, Florida 32816 \\ E-mail: vesko@odalab.ucf.edu
}

\author{
Ravi Vijaya Satya \\ Guy Schiavone \\ University of Central Florida \\ Department of Electrical \\ and Computer Engineering \\ Institute for Simulation and Training \\ Orlando, Florida 32816
}

\section{Eric Clarkson}

University of Arizona

Optical Sciences Center

Tucson, Arizona 87719

\author{
Jannick Rolland, MEMBER SPIE \\ University of Central Florida \\ School of Optics \\ Center for Research and Education in \\ Optics and Lasers \\ Florida Photonics Center of Excellence \\ Institute for Simulation and Training \\ Orlando, Florida 32816
}

\begin{abstract}
A generalized nonparaxial theoretical framework based on the scalar diffraction theory is developed to describe the propagation of an optical field through a linear optical system with quasi-monochromatic spatially incoherent illumination. Software implementation of this theoretical framework on single and multiple processor platforms was developed and simulated results of the imaging process through optical aberration-corrected optics are presented for both in-focus and out-offocus imaging, validating the first-order nonparaxial model. () 2004 Society of Photo-Optical Instrumentation Engineers. [DOI: 10.1117/1.1751401]
\end{abstract}

Subject terms: Huygen's principle; physical optics; scalar diffraction theory; waves; propagation.

Paper IE-OE5 received Oct. 17, 2003; revised manuscript received Mar. 8, 2004; accepted for publication Mar. 9, 2004.

\section{Introduction}

Many imaging scenarios from microscopy to space optics can be well modeled with ray-tracing theory. Some exceptions to that rule include modeling propagation of optical waves in nonlinear media or accounting for coherent or partially coherent light propagation occurring in optical fibers, media with microscopic refractive index variations, and integrated optical components and systems, for example. A good model to describe propagation of light in such systems is the finite-element beam propagation method. ${ }^{1}$ Another approach to modeling the propagation of optical waves in an optical system, Gaussian beam decomposition, is generally applicable in the case where the slowly varying envelope approximation holds. Such an approach is often used to model laser beams or optical field propagation through bulk optics. ${ }^{2}$ Finally, for linear shiftinvariant systems, imaging can be modeled using Fourier transform methods, where the final image is obtained as a convolution of the object with a transfer function. ${ }^{3}$

The application of the scalar diffraction theory to imaging with quasi-monochromatic incoherent light fields presented in this paper was motivated by the domain of optically created special effects, specifically as it applies to propagating an optical field through non-shift-invariant op- tical phase plates strategically positioned within the optics utilized to create the desired special effects. Furthermore, the imaging system may have a large field of view (FOV) as in live image capture. ${ }^{4,5}$ It is beyond the scope of this paper to detail such an imaging system for special effects or demonstrate the creation of special effects that also require optimization of optical texture plates. We shall, however, present a nonparaxial imaging approach that will be essential in future work related to modeling image formation through such phase plates. The modeling approach will also be broadly applicable to non-shift-invariant imaging systems, beyond that of creating special effects.

Note that a system may be non-shift-invariant and optical aberration free. Such a case may be encountered in the creation of optical special effects, where the non-shiftinvariance is created by the texture plates and not the optical aberrations of the imaging optics. However, optical aberrations may contribute to non-shift-invariant optical imaging if optical aberrations of the imaging optics are not fully balanced across the entire FOV. For the live image capture application, the imaging optics should be designed to be distortion free and well corrected to the extent possible for optical aberrations, given that the optics may be utilized with or without the phase plates. Also, if a phase 
Shaoulov et al.: Model of wide-angle optical field propagation ...

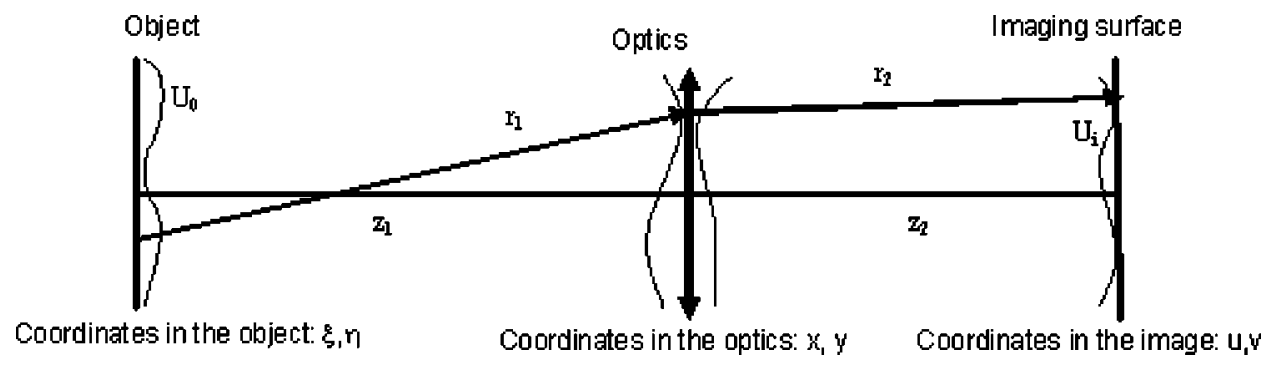

Fig. 1 Layout of an optical system consisting of an object illuminated with a spatially incoherent quasi-monochromatic light field of complex amplitude $U_{o}$, an aberration-free optics, and an arbitrary imaging plane.

plate is utilized, the imaging optics will be used slightly out of focus, therefore the main challenge to this problem is not to model imaging through an aberrated optics, but rather to correctly model first-order wide-FOV imaging, in or out of focus, and to have a modeling approach that in the future will naturally enable insertion of an optical phase plate anywhere within the optics. If a non-shift-invariant texture phase plate is located along the optical path, a non-shiftinvariant imaging model is required regardless of the fact that the imaging optics may be diffraction limited.

The ray-tracing approach was first implemented in early stages of our investigation, however, the application of this approach was limited by the assumption of spatial incoherence everywhere along the optical path and failed to provide a theoretical model describing the behavior of an optical phase plate. ${ }^{5}$ Furthermore, because phase plates are non-shift-invariant, the final image cannot be obtained as a simple convolution. Thus, the use of Fourier-transformbased algorithms is not applicable. Specifically, there is no advantage to using Fresnel diffraction theory and the associated approximations and simplifications that are conventionally used to introduce Fourier transforms as part of the imaging process. ${ }^{3}$ In practice, for very specific image formation problems, it would be interesting to compare various modeling approaches with their associated approximations. Moreover, because the structure of the texture plates is rough, neither Gaussian beam decomposition algorithms, nor stationary scattering techniques such as the HarveyShack technique, are applicable. ${ }^{6}$ Thus, to model the imaging process through a structure imposing such restrictions, a different approach had to be investigated, and custom software implementation had to be developed.

A key contribution of this paper is the presentation of a generalized nonparaxial theoretical framework based on the Rayleigh-Sommerfeld diffraction formulation enabling the application of the scalar diffraction theory to imaging with quasi-monochromatic incoherent illumination across large FOVs. This generalized framework extends the paraxialimaging framework reviewed in Sec. 2 to the nonparaxial case.

\section{Review of the Theoretical Framework for Quasi-Monochromatic Incoherent Illumination}

Scalar diffraction theory has been previously proposed to model quasi-monochromatic incoherent imaging for describing light propagation through a linear optical system. ${ }^{3}$ Such modeling is based on the classical Rayleigh-
Sommerfeld diffraction integral. Let us consider a linear optical system consisting of an object, an aberration-free optics, and an imaging surface (e.g., a plane is a special case), as shown in Fig. 1. If we denote the local coordinate systems in the object, the exit pupil, and the image as $[\xi ; \eta]$, $[x ; y]$, and $[u ; v]$, respectively, and the optical field in the object plane by its complex amplitude $U_{o}(\xi ; \eta)$, then the complex amplitude in the imaging plane $U_{i}(u ; v)$ will be expressed as

$U_{i}(u, v)=\int_{-\infty}^{\infty} \int_{-\infty}^{\infty} \mathrm{d} \xi \mathrm{d} \eta U_{o}(\xi, \eta) h(u, v ; \xi, \eta)$,

where $h(u, v ; \xi, \eta)$ is the transfer function of the investigated system for a point $[u, v]$ given $[\xi, \eta]{ }^{3}$ Furthermore, to express the irradiance distribution in the image, we must consider the statistical properties of the light. By assuming ergodicity of the statistical imaging process and applying Fubini's theorem, ${ }^{7}$ the irradiance distribution in the image can be further written as

$$
\begin{aligned}
\left\langle I_{i}\right\rangle(u, v)= & \int_{-\infty}^{\infty} \iiint\left\langle U_{o}(\xi, \eta) U_{o}^{*}\left(\xi^{\prime}, \eta^{\prime}\right)\right\rangle \\
& \times h(u, v ; \xi, \eta) h^{*}\left(u, v ; \xi^{\prime}, \eta^{\prime}\right) \mathrm{d} \xi \mathrm{d} \eta \mathrm{d} \xi^{\prime} \mathrm{d} \eta^{\prime},
\end{aligned}
$$

where the brackets in the left-hand-side term denote the mean over time and the brackets on the right-hand side denote ensemble average. ${ }^{8}$ Finally, assuming quasimonochronatic spatially incoherent illumination we obtain

$$
\begin{aligned}
\left\langle U_{o}(\xi, \eta) U_{o}^{*}\left(\xi^{\prime}, \eta^{\prime}\right)\right\rangle & =\left\langle\left|U_{o}(\xi, \eta)\right|^{2}\right\rangle \delta\left(\xi-\xi^{\prime}\right) \delta\left(\eta-\eta^{\prime}\right) \\
& \equiv\left\langle I_{o}(\xi, \eta) \delta\left(\xi-\xi^{\prime}\right) \delta\left(\eta-\eta^{\prime}\right)\right\rangle,
\end{aligned}
$$

and thus Eqs. (2) and (3) yields

$\left\langle I_{i}(u, v)\right\rangle=\int_{-\infty}^{\infty} \int\left\langle I_{o}(\xi, \eta)\right\rangle|h(u, v ; \xi, \eta)|^{2} \mathrm{~d} \xi \mathrm{d} \eta$.

Thus, Eq. (4) provides the relationship between the irradiance distribution in the image and the irradiance distribution in the object illuminated with quasi-monochromatic and spatially incoherent light under the assumption of ergodicity of the imaging process. Equation (4) is similar to Gaskill's ${ }^{9}$ Eq. (11.82). It simply differs by taking into ac- 
count the statistical properties of the light. Importantly, the expression for the transfer function must be formally established to apply this framework to different imaging configurations (e.g., paraxial; nonparaxial aberration free; nonparaxial combined with optical aberrations; any of these with defocus given that defocus is best considered as a first-order imaging property).

\section{Review of the Application of the Paraxial Framework to Plane-to-Plane and Plane- to-Curve Imaging Configurations}

In the special case of a system consisting of free-space propagation, followed by propagation through an aberration-free optics of focal length $f$, and consequent propagation through free space, the paraxial transfer function based on applying the Rayleigh-Sommerfeld formula is given by

$$
\begin{aligned}
h(u, v ; \xi, \eta)= & \int_{-\infty}^{\infty} \int_{-\infty}^{\infty} \mathrm{d} x \mathrm{~d} y P(x, y)\left[\frac{\exp \left(j k r_{1}\right)}{j \lambda r_{1}} \cos \theta_{1}\right] \\
& \times\left\{\exp \left[-j \frac{k}{2 f}\left(x^{2}+y^{2}\right)\right]\right\} \\
& \times\left[\frac{\exp \left(j k r_{2}\right)}{j \lambda r_{2}} \cos \theta_{2}\right]
\end{aligned}
$$

where $k$ is the wave number, $\lambda$ is the central wavelength, $\cos \theta_{1}$ and $\cos \theta_{2}$ are obliquity factors, and $P(x, y)$ is a pupil function equal to one inside the pupil and zero everywhere else. In Eq. (5), $r_{1}$ and $r_{2}$ are defined as

$r_{1}=\left[(x-\xi)^{2}+(y-\eta)^{2}+z_{1}^{2}\right]^{1 / 2}$,

$r_{2}=\left[(u-x)^{2}+(v-y)^{2}+z_{2}^{2}\right]^{1 / 2}$.

The obliquity factors occur by considering the geometry of parallel object-to-pupil and pupil-to-image plane-toplane imaging. Thus, one can infer that this expression would hold some validity in the nonparaxial region once the parabolic approximation to the optics transfer function is replaced by the exact expression. We shall demonstrate in Sec. 4 that such inference fails. Equation (5) is quite similar to the framework presented in Sec. 10-6 in Gaskill, ${ }^{9}$ however, it has been made formally a function of four variables, thus allowing to model non-shift-invariant systems.

Furthermore, from the theory of first-order imaging properties, it is known that even for perfectly stigmatic imaging (i.e., aberration-free imaging), the image is not distributed on a plane surface but rather on a curved surface, called the Petzval surface, as shown in Fig. 2. Without loss of generality in illustrating the Petzval surface, Fig. 2 depicts the case of an object at optical infinity. ${ }^{10,11}$ In this case, the surface can be shown to describe a portion of a sphere. If the object is located a finite distance from the optics, the surface shape can be computed using first order imaging equations. ${ }^{12,13}$ Thus, a next step in the formal representation of the basic framework set in Eq. (5) is to consider a plane-to-Petzval-surface imaging process to fully validate that sharp imaging occurs on the predicted curved surface. If field curvature imposed by Petzval curvature is

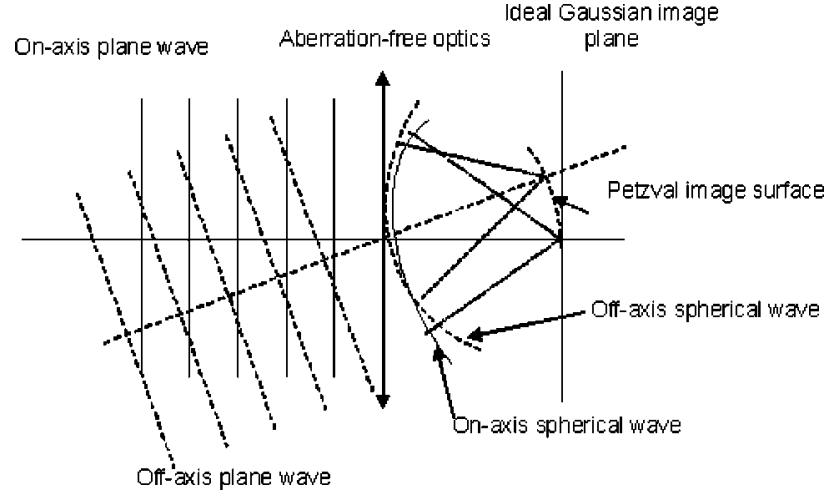

Fig. 2 Illustration of stigmatic imaging on a curve surface for an object located at optical infinity.

severe, as occurrs if the optics is not specifically designed with multiple elements to flatten the field and is imaging large FOVs, the image formed on a plane will be extremely blurred in intensity as one goes up in the FOV along a plane. Also in a simulation with a limited dynamic range (i.e., 0 to 255 gray levels) to represent intensity variations, most of the image will appear to fall within the lowest levels of the gray scale, thus making it difficult to assess the validity of the imaging process, even simply according to its first order imaging properties. We demonstrate such limitations in Sec. 5. Imaging on a curved surface, as opposed to a plane, does not signify that we ignore the Petzval curvature. To the opposite, it signifies that we take it into account in the validation of first-order imaging properties. Such a choice is a necessary step in the validation procedure. Thus the expression for $r_{2}$ in Eq. (6) was modified to

$r_{2}^{\prime}=\left[(u-x)^{2}+(v-y)^{2}+z_{2}^{\prime 2}\right]^{1 / 2}$,

where $z_{2}^{\prime}$ is calculated from the Descartes first-order imaging equation, ${ }^{12,13}$ given $z_{1}$ separately for each set of points $[\xi ; \eta]$ and $[u ; v]$.

\section{Generalized Nonparaxial Theoretical Framework Based on the Scalar Diffraction Theory}

Paraxial imaging is generally rigorously defined for infinitely small object sizes and small angles of incidence on optical elements. Thus, any real imaging scenario, unless on axis only, usually violates the paraxial approximation. In imaging, one then often distinguishes between paraxial, quasi-paraxial imaging [i.e., which applies for FOVs $<10 \mathrm{deg}$ and an optics of an $F$-number $(F \#)$ of 10 or more], and nonparaxial (i.e., FOV $>10$ degrees or an optics of $F \#$ less than 10). Nonparaxial imaging comprises two components in imaging: first-order properties, and higher order imaging properties. First-order properties assume an aberration-free optics and the ability to model in focus imaging on the Petzval surface, as well as imaging with defocus. Higher order properties include optical aberrations. This paper focuses on the first-order nonparaxial imaging properties for an aberration-free system.

A key to generalize the basic framework for nonparaxial imaging was the realization that the phase transformation 


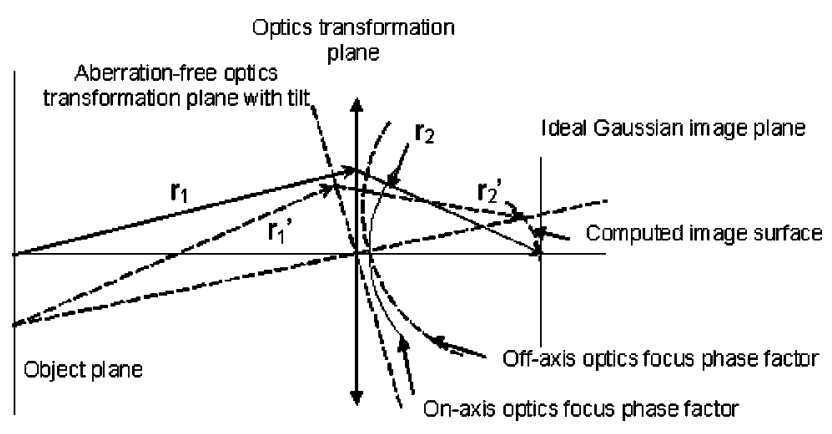

Fig. 3 Generalized nonparaxial imaging framework with the transformation plane normal to the chief ray and tilted with respect to the paraxial transformation plane.

performed by the optics, whether it is the parabolic approximation or the exact form, must be applied on a transformation plane tilted with respect to the optical axis. Equivalently, the transformation must be applied in such a way that it remains normal to the chief ray for each point $[\xi ; \eta]$ in the object, as illustrated in Fig. 3. The physical meaning of the tilted summation plane can be best understood by considering the special case of an extended object at infinity. The wavefront reaching the optics from a point in the FOV is tilted with respect to the optical axis and perpendicular to the chief ray. The lens function is to transform such plane wavefront into a spherical wavefront whose center of curvature lies off-axis on the Petzval surface. Such an imaging property can be accomplished by an optics transformation function that is rotationally symmetric, while not strictly speaking quadratic, with respect to the chief ray. The concept of the tilted plane for summation applies equally well for an object at finite distance from the optics, given that the tilted summation plane is perpendicular to the chief ray in all cases and tangent to the incident wavefront emanating from any object point in the FOV. Therefore the expressions for $r_{1}$ and $r_{2}$ in Eq. (6) were modified to

$$
\begin{aligned}
& r_{1}^{\prime}=\left[\left(x^{\prime}-\xi\right)^{2}+\left(y^{\prime}-\eta\right)^{2}+z_{1}^{\prime 2}\right]^{1 / 2}, \\
& r_{2}^{\prime}=\left[\left(u-x^{\prime}\right)^{2}+\left(v-y^{\prime}\right)^{2}+z_{2}^{\prime 2}\right]^{1 / 2},
\end{aligned}
$$

where $\left[x^{\prime}, y^{\prime}\right]$ are the coordinates in the tilted lens transformation plane. Thus, the transfer function introduced in Eq. (2) can be reduced to

$$
\begin{aligned}
h(u, v ; \xi, \eta)= & -\int_{-\infty}^{\infty} \int_{-\infty}^{\infty} \mathrm{d} x^{\prime} \mathrm{d} y^{\prime} P\left(x^{\prime}, y^{\prime}\right) \\
& \times \frac{\exp \left(j k r_{1}^{\prime}\right) \exp -j k\left\{\left(z_{1}^{\prime}+z_{2}^{\prime}\right)-z_{1}^{\prime}\left[1-\left(x^{\prime 2}+y^{\prime 2}\right) / z_{1}^{\prime 2}\right]^{1 / 2}-z_{2}^{\prime}\left[1-\left(x^{\prime 2}+y^{\prime 2}\right) / z_{2}^{\prime 2}\right]^{1 / 2}\right\} \exp \left(j k r_{2}^{\prime}\right)}{\lambda^{2} r_{1}^{\prime} r_{2}^{\prime}},
\end{aligned}
$$

where $P\left(x^{\prime}, y^{\prime}\right)$ is equal to 1 inside the projection of the aperture on the tilted plane and equal to zero outside, $z_{1}^{\prime}$ and $z_{2}^{\prime}$ are related through the Descartes equation, and the function describing the optics as a focusing element with no aberrations is an exact expression, instead of the parabolic approximation. The lens term in Eq. (9) was derived by considering the dephasing required to be introduced by a converging lens to map a diverging spherical wavefront to a converging spherical wavefront. With this mathematical formulation, the obliquity factors are not necessary given that the summation planes are always perpendicular to the chief rays.

\section{Results}

To fully demonstrate the capability of the model described by Eqs. (7) to (9), we must first choose reasonable values for the parameters of the optical system not only within the quasi-paraxial region, but preferably within the nonparaxial region. Thus, the object was considered located at a distance of $2 f$ away from an $F / 5$ aberration-free optics of 20-mm focal length, enabling testing of both the paraxial and the nonparaxial frameworks for various FOVs.

The mathematical framework was implemented using conventional numerical quadrature techniques (e.g., see Ref. 14). Given the high number of computations involved, the computation time for the simulation of one imaging condition was over 3 weeks on a single-processor platform. Thus, establishing an alternative computational approach was necessary. The next-generation implementation was developed on a Beowulf cluster utilizing 96 dual 1.3-Ghz Athlon processor nodes. ${ }^{15}$ The computation to generate the simulated images was easily spatially partitioned to handle subsections of the object to be imaged. This distribution resulted in implicit load balancing. The time complexity and accuracy in terms of the size of the matrix elements, as well as the scalability on a parallel platform will be discussed in a follow-up investigation, because it requires an in-depth investigation of various parallel architectures including 32-bit versus 64-bit processors, as well as assessment of performance on symmetric multiprocessing (SMP) and nonuniform memory access (NUMA)-based computing platforms. ${ }^{16}$ With the Beowulf cluster implementation, the computation time was reduced to minutes, which enabled us to further investigate the modeling approach.

The generalized nonparaxial theoretical framework for plane-to-curve imaging was compared to the paraxial or more precisely the quasi-paraxial framework for plane-tocurve imaging reviewed in Sec. 3, by using a 256-graylevel bitmap object filling a 40-deg FOV. Equation (5), which defines the paraxial and quasi-paraxial framework, is thought to be paraxial or quasi-paraxial because of the parabolic approximation to the optics transformation func- 


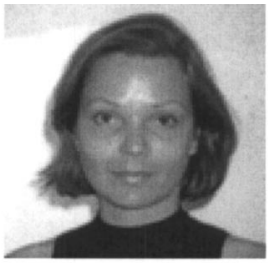

Object

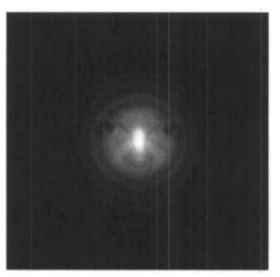

(a)

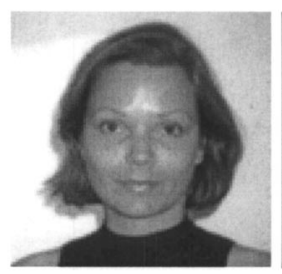

(b)

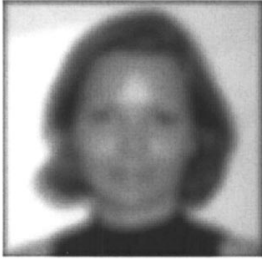

(c)
Fig. 4 Results of imaging a 40-deg FOV object in a $2 f$ imaging configuration for plane-to-curve imaging: (a) paraxial/quasi-paraxial model given by Eq. (2), (b) nonparaxial model given by Eq. (5) for in-focus imaging, (c) same as (b) but with 3-mm out-of-focus imaging.

tion. However, the main reason for this equation to be limited to the quasi-paraxial region is the application of the optics transformation function on a plane perpendicular to the optical axis, as we show in the simulations. Equation (9) addresses both limitations of Eq. (5) by using an exact optics transformation function and applying it in a plane perpendicular to the chief ray for each point in the FOV.

All results presented correspond to plane-to-curve imaging, where the Petzval surface was computed for the geometry provided, to ensure that a lack of a good image or even an image across the full FOV is not a consequence of Petzval curvature. Under realistic imaging conditions with an optimized optics, the Petzval curvature will be moderate, and in the case of plane-to-plane imaging it will cause only slight blur as the image extends further in the FOV. However, because we do not use a field flattener within the optics or other ways (i.e., combination of positive and negative separated optical elements) to reduce the Petzval curvature, the Petzval surface curves severely in the case we considered, and must be accounted for by imaging on its curved surface. In the case where optimized optics with their flattened Petzval curvature and associated optical aberrations is considered, the optics transformation can be built accordingly. The optics transformation is always applied in the exit pupil to model the entire optics via one phase transformation. Furthermore, under all imaging conditions, the images of the face were presented upright for easier comparison, accounting for the optics providing inverted images.

Results shown in Fig. 4(a) demonstrate that the paraxial framework fails for large FOVs, as expected. Only a portion of the face imaged within the quasi-paraxial region is seen sharply. Past the quasi-paraxial region, the image dims so quickly that when displayed on a 0 - to 255-gray-level scale, it is non-perceivable.

To further yield insight into the issue of dynamic range (i.e., relative brightness for various points in the FOV), an object with a small square at the edge was selected as the

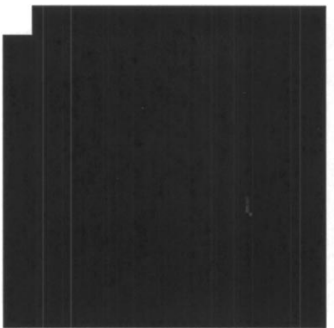

(a)

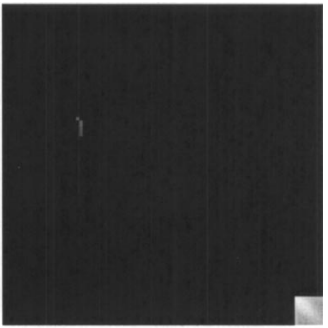

(b)
Fig. 5 Demonstration of the existence of low light levels at the edge of the FOV in the case of the paraxial model and plane-to-curve imaging: (a) object with one white square at the edge of the FOV and (b) image.

object to be imaged as shown in Fig. 5(a). The result from the paraxial framework is demonstrated in Fig. 5(b), where the white square appears as expected but somewhat dimmer. Also, the intensity within the square is nonuniform. In this simple stimulus case, because there is no light intensity anywhere else in the object and the nonuniformities within the square are relatively small, no other part of the object competes for the dynamic range, thus when the image output is scaled from 0 to 255 gray levels, no matter how dim in average the square at the edge is, it will be displayed at gray levels close to 255 .

If we now consider an object with two squares, one in the center and one at the edge, as shown in Fig. 6(a), results from both the paraxial and the nonparaxial frameworks are shown in Figs. 6(b) and 6(c), respectively. Results indicate that using the paraxial framework, the square at the edge now disappears, indicating that it is a lot dimmer than the one in the center. The non-paraxial framework yields imaging of both squares with equal brightness on the curved surface as one expects from an appropriate 1st-order nonparaxial imaging framework.

Results shown in Fig. 4(b) demonstrate that the expanded nonparaxial framework works for large FOVs. Finally, to verify the first-order defocusing imaging properties with the nonparaxial framework, the imaging surface was placed $3 \mathrm{~mm}$ out of focus toward the optics. Results presented in Fig. 4(c) demonstrate that the generalized nonparaxial theoretical framework works for out-of-focus imaging as well.

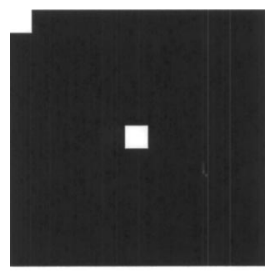

(a)

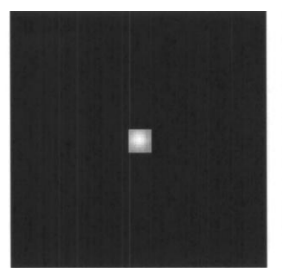

(b)

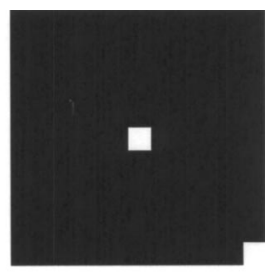

(c)
Fig. 6 Demonstration of the existence of low light levels at the edge of the FOV in the case of the paraxial model and for plane-to-curve imaging: (a) object with one white square in the middle and one white square at the edge, (b) image of (a) using the paraxial model, the square at the edge is extremely dim with respect to that in the center and thus cannot be seen within a limited 255-gray-levels display; and (c) image of (a) using the nonparaxial model. Such an imaging scenario models accurately imaging with a 40-deg FOV. 


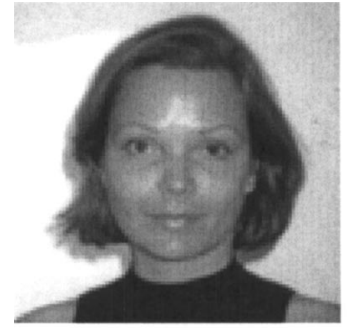

(a)

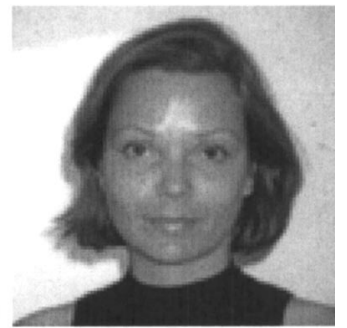

(b)
Fig. 7 Results of the nonparaxial theoretical framework for planeto-curve imaging using (a) no approximation for the optics transformation, except assuming an aberration-free optics, and (b) nevertheless the parabolic optics transformation approximation, which could be thought to be responsible for having the model provided by Eq. (2) fail. Results reveal that the parabolic approximation for the optics transformation leads to negligible effects on overall imaging even for nonparaxial imaging, thus such an approximation could be employed even in some nonparaxial case (i.e., moderate $F$-number) if it was to reduce the complexity of the computations.

Finally, we intend for this paper to bring forth the fact that within the paraxial framework, the parabolic approximation to the optics transformation function can be insignificant compared to the fact that in that regime the optics transformation is applied in a plane perpendicular to the optical axis. Furthermore, if we are correct, the nonparaxial framework would work equally well with or without the parabolic approximation to the optics transformation for a moderate $F$-number, as long as the summation occurs in a plane perpendicular to the chief ray. Thus, we ran a simulation with the nonparaxial equation under both cases of the optics transformation. The results presented in Fig. 7 indicate that the nonparaxial framework works equally well with the parabolic approximation to the optics transformation function and with the exact expression, while results presented in Figs. 4(a) and 4(b) indicated that even when the exact expression of the optics transformation function was used, imaging failed if the optics transformation was not applied in a plane perpendicular to the chief ray.

\section{Conclusions and Future Work}

The principles of scalar diffraction theory were applied to optical imaging of extended objects under quasimonochromatic incoherent illumination. The existing theoretical framework was generalized to nonparaxial in-focus and out-of-focus imaging and results were obtained to verify the generalized framework. In that framework, the optical system was considered linear. In the generalized nonparaxial framework, even the most complex linear systems without any assumptions for shift invariance, can be modeled and analyzed. Finally, this work will be extended in the near future to include higher order aberrations in the optics transformation equation and special effects imaging by including a propagation stage through optical phase plates.

\section{Acknowledgments}

This work was supported in part by the U.S. Army Simulation, Training, and Instrumentation Command (STRICOM). We would like to thank Sergey Polyakov,
Demetrios Christodoulides, and Boris Zeldovich from the School of Optics for stimulating discussions about this work.

\section{References}

1. L. Vincetti, A. Cucinotta, S. Selleri, and M. Zoboli, "Threedimensional finite-element beam propagation method: assessments and developments," J. Opt. Soc. Am. A 17(6), 1124-1131 (2000).

2. M. Montoya-Hernandez, M. Servin, D. Malacara-Hernandez, and G. Paez, "Wavefront fitting using Gaussian functions," Opt. Commun. 163, 259-269 (1999).

3. J. W. Goodman, Introduction to Fourier Optics, 3rd ed., McGrawHill, New York (2000).

4. J. P. Rolland and S. Hylen, "Painting cameras," OPN 10(7), 33-35 (1999).

5. V. Shaoulov, C. Meyer, Y. Argotti, and J. P. Rolland, "Optical phase plates as a creative media for special effects in photography," Proc. SPIE 4442, 112-118 (2001).

6. J.E. Harvey, "Light-scattering characteristics of optical surfaces," Ph.D. dissertation, University of Arizona, Tucson, Arizona (1976).

7. H. L. Royden, Real Analysis, p. 269, MacMillan, New York (1968).

8. J. W. Goodman, Statistical Optics, John Wiley \& Sons, New York (2000).

9. J. Gaskill, Linear Systems, Fourier Transforms and Optics, John Willey \& Sons, New York (1978)

10. G. Slyusarev, Aberration and Optical Design Theory, Adam Hilger Ltd., Bristol (1984).

11. D. Malacara and Z. Malacara, Handbook of Lens Design, Marcel Dekker, New York (1994).

12. P. Mouroulis and J. Macdonald, Geometrical Optics and Optical Design, Oxford Press, New York (1996).

13. J. P. Rolland, V. Shaoulov, and F. J. Gonzalez, "The art of back-of-the envelope paraxial raytracing," IEEE Trans. Edu. 44(4), 365-372 (2001).

14. R. L. Burden and J. Faires, Numerical Analysis, 7th ed., Brooks Cole (7th edition), Pacific Grove, California (2000).

15. D. Spector, Building Linux Cluster, O'Reilly, Sebastopol (July 2000); http://www.beowulf-underground.org/.

16. J. Dongarra, The Sourcebook of Parallel Computing, Morgan Kaufmann, San Francisco, California (2002).

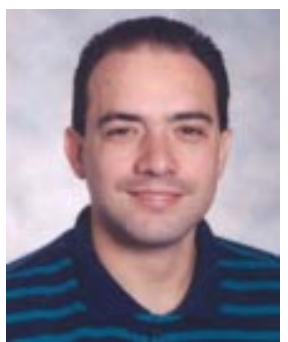

Vesselin Shaoulov is a PhD candidate at the School of Optics and Center for Research and Education in Optics and Lasers (SoO/CREOL) at the University of Central Florida (UCF). In 1994 he received his BS in physics and in 1996 his MS in physics, both from the Sofia University "St. Kliment Ohridsky" in Sofia, Bulgaria, and in 2000 he received his $\mathrm{MS}$ in optics from $\mathrm{SoO} /$ CREOL. He joined the Optical Design and Applications Laboratory at SoO/CREOL/ UCF in 1998 as a graduate student in optics and began work as a $\mathrm{PhD}$ candidate in 2000 . He is currently working in the area of design and assessment of compact optical systems, illumination design, and theoretical modeling of light propagation through optical systems and textures. He has authored and co-authored more than 10 peer-reviewed publications and conference proceeding papers and a patent related to the same topics.

Ravi Vijaya Satya Biography and photograph not available.

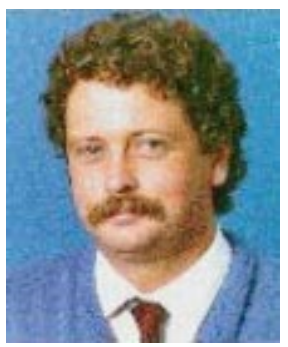

Guy Schiavone is an assistant professor with the Computer Engineering Department at the University of Central Florida (UCF). He has served as principal investigator $(\mathrm{PI})$ and co-PI on several research projects at UCF in various areas of modeling and simulation and computer engineering. Dr. Schiavone held previous positions as visiting assistant professor in the UCF Computer Science Department, senior research scientist at University of Michigan, and research scientist at Institute for Simulation and Training. He has led projects and published in the areas of electromagnetics, distributed and parallel processing, terrain databases, 3-D modeling, analysis and visualization, and interoperability in distributed 
simulations for training. He received the $\mathrm{PhD}$ in engineering science from Dartmouth College in 1994 and the BEEE from Youngstown State University in 1989.

Eric Clarkson Biography and photograph not available.

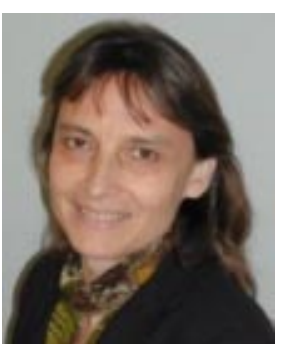

Jannick Rolland is an associate professor of optics, computer science, electrical engineering, and modeling and simulation at the University of Central Florida (UCF). She received a diploma from the Ecole Superieure D'Optique in Orsay, France, in 1984 , and her PhD in optical science from the University of Arizona in 1990. She then joined the Department of Computer Science at the University of North Carolina at Chapel Hill (UNC-CH) as a postdoctoral student to conduct research in optical design for 3-D medical visu- alization. She was appointed to the research faculty at UNC in 1992 and headed the Vision Research Group from 1992 to 1996. She teaches graduate courses in optical system design and leads research in novel optical instruments for 3-D visualization and imaging. She holds four patents, has written 6 book chapters, and has authored over 35 peer reviewed publications related to optical design, augmented reality, vision, and image quality assessment for medical imaging. She has authored and co-authored over 75 other publications related to the same topics. Dr. Rolland has been an associate editor of Presence (MIT Press) since 1996 and an Associate Editor of Optical Engineering since 1999. She was the UCF distinguished professor for the year 2001 for the UCF centers and institutes and is a fellow of the OSA. 